\title{
CORRECTIONS TO \\ “ON SEQUENTIAL DECISION PROBLEMS WITH \\ DELAYED OBSERVATIONS"
}

\author{
YUKIO SUZUKI
}

The author is indebted to Professor K. Miyasawa of Tokyo University for drawing his attention to the incorrect statements in theorem 2.3 and its proof of the above titled paper (Ann. Inst. Statist. Math., 18 (1966), 229-267). In the theorem "but not necessarily convex" should be deleted and in its proof "The statement that $\Xi_{j}^{*} j=0, \cdots, N$ is not necessarily convex will be clarified in the sections 3 and 4 . This is a typical character due to the existence of delayed observations." should be deleted. Then the theorem and its proof are correct.

The author now considers that it is suitable to add the following remark after the rectified theorem and its proof:

Remark 2.2. A typical character of $z^{*} j=0, \cdots, N$ due to the existence of delayed observations will be clarified by Proposition II and its justification in the sections 3 and 4 .

Other corrections :

(1) $\tau_{j+m, \xi}^{*}(x)$ in expressions (1.16), (1.17) and (1.18) should read $\tau_{j, s}^{*}(x)$.

(2) $\underline{H}$ in expression (4.50) should read $\underline{H}_{n}$.

(3) $H_{n}$ in expression (4.51) should read $\underline{H}_{n}$. 